Projets

de paysage

\section{Projets de paysage}

Revue scientifique sur la conception et l'aménagement de l'espace

$16 \mid 2017$

Arbres et paysages

\title{
Végétation des espaces boisés et paysage urbain
}

Le cas d'une une ville moyenne

Vegetation of Wooded Areas and the Urban Landscape - The Case of an AverageSized City

Francesca Di Pietro et Lotfi Mehdi

\section{OpenEdition}

\section{Journals}

Édition électronique

URL : http://journals.openedition.org/paysage/5621

DOI : 10.4000/paysage.5621

ISSN : 1969-6124

\section{Éditeur :}

École nationale supérieure du paysage de Versailles-Marseille, Institut national des sciences appliquées Centre Val de Loire - École de la nature et du paysage, École nationale supérieure d'architecture et de paysage de Bordeaux, École nationale supérieure d'architecture et de paysage de Lille, Agrocampus Angers

\section{Référence électronique}

Francesca Di Pietro et Lotfı Mehdi, « Végétation des espaces boisés et paysage urbain », Projets de paysage [En ligne], 16 | 2017, mis en ligne le 07 juillet 2017, consulté le 30 avril 2020. URL : http:// journals.openedition.org/paysage/5621 ; DOI : https://doi.org/10.4000/paysage.5621

Ce document a été généré automatiquement le 30 avril 2020.

Projets de paysage 


\section{Végétation des espaces boisés et paysage urbain}

Le cas d'une une ville moyenne

Vegetation of Wooded Areas and the Urban Landscape - The Case of an AverageSized City

Francesca Di Pietro et Lotfi Mehdi

\section{La végétation des espaces boisés urbains : quelle relation avec le paysage?}

1 Dans les paysages urbains les espaces boisés recouvrent un large panel de situations écologiques et foncières, impliquant ou pas l'ouverture au public. Du point de vue morphologique, nous pouvons distinguer deux types d'espaces boisés : d'une part, les arbres des rues (arbres isolés ou en alignement) et la végétation associée (végétation des pieds d'arbres); d'autre part, les peuplements arborés inclus dans la tache urbaine : les forêts urbaines.

2 Les arbres des rues et la végétation qui y est associée, la végétation des pieds d'arbres, font l'objet d'études spécifiques dans plusieurs grandes villes: des recensements à Hong Kong (Jim, 1998) et New York (Cowett \& Bassuk, 2014), des approches historiques à Tunis (Bennour-Azooz et al., 2012). Une recherche sur l'aménagement des pieds d'arbres à Paris met en évidence l'évolution de ces microterritoires : si les trottoirs plantés existent depuis la fin du xvIII ${ }^{\mathrm{e}}$ siècle, à côté du pied d'arbre constitué de terre sans protection, le trio indissociable trottoir-fosse-grille a été diffusé au XIX ${ }^{\mathrm{e}}$ siècle dans un objectif d'hygiène, de confort et d'esthétique, afin de faciliter et de sécuriser le cheminement piétonnier tout en permettant l'installation des arbres. Récemment les pieds d'arbres stabilisés, enherbés, puis jardinés, remplacent en partie les grilles "haussmanniennes» et la diversité des usages détermine le type de pied d'arbre: enherbé dans une rue peu fréquentée ; fosse remplie d'un revêtement stabilisé, avec ou sans grille, dans une rue commerçante; grille haussmannienne dans les zones 
touristiques (Pellegrini, 2012). En général la plantation des arbres dans les sols des villes, revêtus de goudron, et leur gestion, posent des difficultés spécifiques aux services techniques des villes, en raison du système racinaire (Mullaney et al., 2015) mais aussi de la fragilité des arbres vis-à-vis des organismes ravageurs (Subburayalu et Sydnor, 2012). Outre ces difficultés d'ordre technique, la gestion urbaine des arbres pose, aux services municipaux, des difficultés institutionnelles, liées à un problème de coordination entre différents services municipaux, dont les aires d'intervention se chevauchent (Haddad, 1997 ; Pellegrini, 2012). Cette gestion pose aussi un problème d'ordre social : les arbres en ville et la végétation des rues, entretenue ou spontanée, peuvent constituer un sujet de plainte extrêmement récurrent de la part des habitants (Tollis, 2013) ou bien être reconnus dans leurs différentes composantes végétales et être appréciés par les habitants (Weber et al., 2014b). La perception des arbres en ville dépend du type de tissu urbain: en milieu fortement urbanisé, l'arbre des rues représente l'essentiel du patrimoine végétal et prend un caractère symbolique important, alors que dans les zones pavillonnaires, où il existe une végétation privée, l'attachement aux arbres "publics» est moindre. Cette perception des arbres en ville dépend également des usages urbains: la population est animée de sentiments contradictoires selon qu'elle représente passants, riverains ou automobilistes (Haddad, 1997). Une tension existe entre les différents rôles qu'on attribue aux arbres d'alignement en ville (symbolique, identitaire, social, utilitaire, etc.) ; cette tension explique que $70 \%$ du patrimoine arboré est représenté par un petit nombre d'essences (2 à 8 espèces : Haddad, 1997). Malgré cela, les rues urbaines peuvent héberger une très grande variété d'espèces animales et végétales qui colonisent les microsites existant ou utilisent, pour se déplacer, ces corridors routiers comme parties de réseaux d'habitats urbains (Säumel et al., 2015). Ces corridors urbains végétalisés, généralement discontinus, «en pas japonais », peuvent réduire l'isolement des habitats semi-naturels en ville (White et al., 2005), où des populations végétales sont affectées par la fragmentation de leur habitat (Dubois et Cheptou, 2016) mais aussi par la colonisation à partir d'habitats proches ou plus éloignés (Dornier et al., 2011). Cependant une autre étude met en relief l'homogénéisation de la végétation spontanée des pieds d'arbres dans le monde (Wittig et Becker, 2010). Deux synthèses bibliographiques soulignent la diversité des services écosystémiques fournis par ce type de végétation urbaine (Mullaney et al., 2015 ; Säumel et al., 2015) ; les arbres en milieu urbain ont des effets sur l'érosion hydrique (Armson et al., 2013) et sur l'ensoleillement (Bajsanski et al., 2016) ainsi que des effets complexes sur la pollution atmosphérique (McPherson et Simpson, 1999 ; Weber et al., 2014a ; Grote et al., 2016).

3 En ce qui concerne les forêts urbaines, qui comprennent les espaces verts publics incluant des bois, elles peuvent représenter une partie considérable des espaces urbains : $20 \%$ des espaces urbains en moyenne en Suède, où les bois urbains et périurbains couvrent une superficie supérieure à celle des forêts protégées; ceci en particulier dans les communes périurbaines qui sont supposées connaître un accroissement démographique pouvant conduire à la fragmentation des forêts urbaines (Hedblom et Söderström, 2008). En zone périurbaine, ces forêts peuvent représenter des habitats reliant les îlots boisés urbains et les zones forestières rurales de plus grande étendue (Pirnat, 2000). Une littérature scientifique en émergence souligne le fait que les espaces boisés urbains abritent une part importante des espèces trouvées dans les espaces environnant les villes, y compris des espèces vulnérables (Alvey, 2006); les îlots boisés confortent la biodiversité urbaine en ce qui concerne plusieurs 
communautés animales (Croci et al., 2008). Concernant la végétation des forêts urbaines, plusieurs études soulignent l'influence du gradient d'urbanisation, à l'échelle de la tache urbaine, et celle de la structure interne des bois (effet lisière), à l'échelle de l'habitat, sur les caractéristiques fonctionnelles des communautés (Godefroid et Koedam, 2003 ; Vallet et al., 2010). À une échelle intermédiaire entre la tache urbaine et l'habitat, celle du paysage urbain (Clergeau et al., 2006), d'autres études montrent l'influence de l'occupation du sol adjacente aux îlots boisés, et de la dimension de ces îlots, sur la composition et la richesse spécifique des communautés végétales, en particulier celles des sous-bois périurbains (Guirado et al., 2006). La végétation forestière le long d'un gradient d'urbanisation a également été étudiée en tant que témoin des effets du changement climatique et de l'urbanisation passée (Carreiro et Tripler, 2005). Plusieurs études font par ailleurs état des effets positifs des forêts urbaines sur la santé humaine (effets sur des patients en dépression nerveuse: Sonntag-Öström et al., 2015), le bien-être (à travers le silence : Skärbäck, 2007) et les pratiques sportives des habitants (Qvistrom, 2016). À ces deux habitats, arbres des rues et forêts urbaines, une synthèse bibliographique récente sur les arbres en milieu périurbain ajoute les bois résiduels et les espaces résidentiels, privés (Nitoslawski et al., 2016).

4 Dans l'ensemble de ces situations, les recherches montrent que la présence d'arbres, qu'il s'agisse de plantations ou d'îlots boisés semi-naturels, s'accompagne d'une végétation ligneuse et herbacée spontanée, qui contribue considérablement à la biodiversité urbaine. L'objectif de cet article est de faire état des recherches conduites sur la végétation abritée par les espaces boisés urbains, et ses relations avec le paysage urbain. Nous nous intéressons plus particulièrement au sous-bois car, contrairement aux arbres, le sous-bois abrite généralement des espèces spontanées, plus susceptibles que les arbres eux-mêmes de refléter les processus de colonisation végétale liés à la diversité du paysage et d'enrichir la biodiversité urbaine. Nous faisons l'hypothèse que cette végétation est liée au paysage urbain, et en particulier à l'un de ses principaux descripteurs : l'occupation du sol. L'influence du paysage urbain, d'une part, et de la structure interne aux habitats boisés (effet lisière), d'autre part, a été plus particulièrement étudiée.

5 Nous soulignons en outre que, à quelques exceptions près, la plupart des travaux conduits sur la végétation arborée urbaine, et en particulier les travaux sur les arbres des rues, ont concerné les villes de grande taille, comprenant au moins un million d'habitants (Berlin, Cologne, Bruxelles, Paris, Barcelone, etc.). Les villes de taille moyenne ont été peu étudiées; c'est toutefois dans ces villes que l'expansion urbaine, entendue comme la conjugaison de la croissance démographique et de l'étalement urbain, est la plus prononcée (Santamaria, 2000 ; Brun, 2015). Notre site d'étude est une zone urbaine de taille moyenne, l'agglomération de Tours, qui compte environ 300000 habitants dans le périmètre administratif de la communauté d'agglomération.

\section{Méthodologie d'échantillonnage}

6 La végétation des espaces publics boisés a été étudiée dans l'agglomération de Tours, plus précisément dans sept communes constituant la tache urbaine, parmi les quatorze communes qui formaient la communauté d'agglomération, dans le cadre d'une thèse de doctorat (Mehdi, 2010) et selon un protocole inspiré d'une autre recherche conduite sur 
ce sujet (Vallet, 2009). Treize espaces publics boisés d'une superficie supérieure à un hectare ont été sélectionnés; il s'agit de bois situés dans des conditions stationnelles comparables: bois semi-naturels pour la plupart (communication personnelle des services techniques de la ville de Tours), sur sols bruns lessivés, en terrain plat, avec une densité arbustive moyenne et une faible fréquentation (figure 1).

Figure 1. Localisation des espaces boisés étudiés

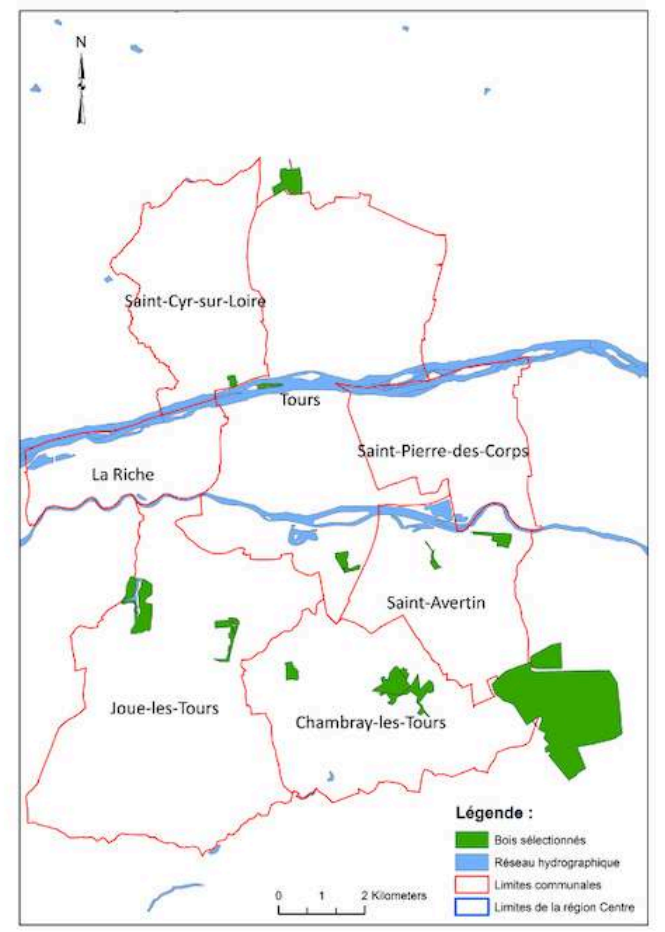

Source : Lotfi Mehdi, 2010.

\section{Méthodologie d'analyse de la végétation}

Dans ces bois, des placettes d'égale surface $\left(30 \mathrm{~m}^{2}\right)$, localisées de façon à maximiser leur éloignement les unes des autres, ont été placées dans la zone centrale et en lisière des bois (figure 2). Dans chaque bois ont été positionnés cinq quadrats de $5 \times 6$ mètres en zone centrale (à une distance minimale de 6 mètres à partir du bord du bois) et cinq quadrats de 3 x 10 mètres dans la lisière interne, pour un total de dix relevés par bois, soit 130 relevés en tout. Les espèces végétales de chacune des trois strates (herbacée : < $1 \mathrm{~m}$, arbustive : entre 1 et $7 \mathrm{~m}$, arborée : $>7 \mathrm{~m}$ ) ont été relevées, ainsi que leur abondance, estimée de 1 à 5 . Ici nous ne traitons que la strate herbacée (sous-bois), la moins liée à des actions intentionnelles et la plus susceptible de traduire les effets du paysage (Mehdi, 2010). 
Figure 2. Un exemple de sous-bois étudié

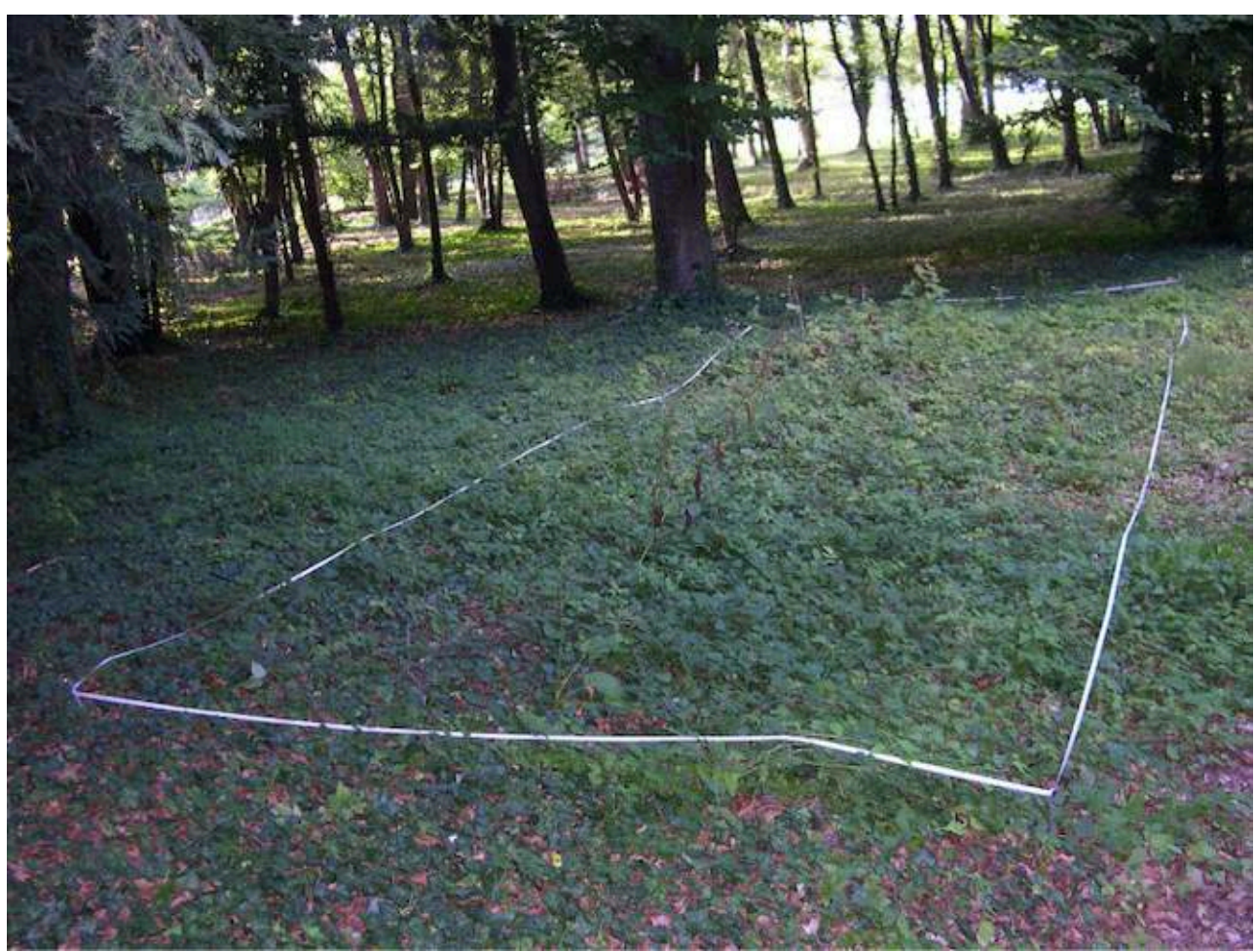

Ici la délimitation d'une placette de lisière.

Source : Lotfi Mehdi, 2010.

Des analyses statistiques multivariées - analyse en composantes principales (ACP), analyse factorielle des correspondances (AFC) et analyse canonique des correspondances ACC - ont été utilisées pour caractériser la variabilité de la composition botanique des sous-bois et analyser sa relation avec les variables de paysage (logiciels utilisés : XLSTAT et Canoco).

\section{Méthodologie d'analyse du paysage urbain}

9 Entre l'échelle locale de l'habitat, et celle plus globale de la tache urbaine, le paysage urbain a été défini en écologie urbaine comme l'échelle intermédiaire du quartier, identifiant un tissu urbain spécifique constitué d'un agencement particulier de bâti, d'espaces verts et de voirie, qui diffère généralement entre le centre et la périphérie (Clergeau et al., 2006). Dans notre étude, le paysage urbain a été étudié principalement sur la base de l'occupation du sol adjacente aux espaces verts boisés, observée dans des zones-tampons de trois rayons autour du centre de l'espace vert : $100 \mathrm{~m}, 500 \mathrm{~m}$ (Kong et al., 2005 ; Kong et Nakagoshi, 2006 ; Vallet, 2009), et d'un rayon supérieur : 750 m. Dans ces zones-tampons nous avons mesuré la proportion de chacune des quatre grandes classes d'occupation du sol: surface urbanisée (bâtie et non bâtie), surface agricole, surface semi-naturelle (notamment forestière), zone hydrographique. En outre, nous avons pris en considération des descripteurs de la position de l'espace boisé dans la tache urbaine (distances au centre-ville, à l'espace vert et au cours d'eau les plus proches), et des descripteurs locaux des bois étudiés : la situation interne au bois (placette centrale ou en lisière), la surface du bois et son âge approximatif. Concernant 
cette dernière variable, l'ancienneté des plantations a été estimée par les gestionnaires et classée en deux groupes: récente (24-35 ans) ou ancienne (plus de 100 ans). L'ensemble des descripteurs de l'espace urbain utilisés sont présentés et hiérarchisés par niveau d'organisation dans le tableau 1.

Tableau 1. Les descripteurs de l'espace urbain utilisés

\begin{tabular}{|l|l|l|}
\hline Niveau d'organisation & Variable & Modalités \\
\hline \multirow{3}{*}{ Tache urbaine } & Distance au centre-ville & Variable quantitative \\
\cline { 2 - 3 } & Distances à l'espace vert le plus proche & Variable quantitative \\
\cline { 2 - 4 } & Distances au cours d'eau le plus proche & Variable quantitative \\
\hline \multirow{2}{*}{ Paysage urbain } & Occupation du sol adjacente au bois & Quatre classes dans trois rayons \\
\hline \multirow{3}{*}{ Habitat urbain } & Surface du bois & Variable quantitative \\
\cline { 2 - 4 } & Âge du bois & Deux classes : récent / ancien \\
\cline { 2 - 4 } & Position interne au bois & Deux classes : central / en lisière \\
\hline
\end{tabular}

10 Concernant la structure spatiale urbaine, l'occupation du sol adjacente aux bois a été analysée par une ACP sur la composition d'occupation du sol adjacente dans les trois rayons considérés. Cette analyse montre que le rayon de $500 \mathrm{~m}$ est le plus discriminant ; les deux classes d'occupation du sol les plus discriminantes sont les surfaces urbanisées et les surfaces agricoles. Cette étude permet de distinguer des bois urbains " centraux », principalement entourés de surfaces urbanisées ( 9 bois sur 13) et des bois urbains " périphériques », entourés d'une forte proportion de surfaces agricoles ( 4 bois sur 13). Nous soulignons ici la diversité de l'occupation du sol de la tache urbaine, impliquant, en zone périurbaine, une proportion non négligeable de surfaces non urbanisées, notamment agricoles.

\section{Lien entre la végétation des espaces boisés et le paysage urbain}

11 Sur l'ensemble des treize espaces boisés étudiés, 219 espèces ont été relevées dans le sous-bois, les strates arborées et arbustives étant constituées principalement d'érable champêtre (Acer campestre L.), de chêne rouvre ou chêne sessile (Quercus petraea [Matt.] Liebl.) et de châtaignier (Castanea sativa Mill.). La liste des espèces trouvées dans les sous-bois étudiés est disponible dans le tableau 2 que vous trouverez en annexe.

En ce qui concerne la variabilité de la végétation des bois par rapport au paysage urbain, nous constatons que la richesse spécifique des sous-bois et sa modulation par l'abondance (indice de Shannon) n'obéissent pas à un gradient d'urbanisation : les bois périphériques, très étendus et entourés majoritairement de cultures, ne sont pas plus diversifiés que les bois centraux (figure 3). En ce qui concerne la variabilité de la végétation par rapport à la structure spatiale interne aux bois, nous observons que la 
diversité spécifique de la lisière est supérieure à celles de la zone centrale dans la plupart des cas ( 9 bois sur 13), et à peine inférieure dans les autres cas (figure 3). Ceci bien que les communautés de sous-bois centrales et de lisière soient très similaires (selon l'indice de Dice, ou de Sorensen, calculé sur les données des espèces en présence/ absence ; Mehdi, 2010).

Figure 3. Diversité spécifique (indice de Shannon) du sous-bois des espaces publics urbains étudiés

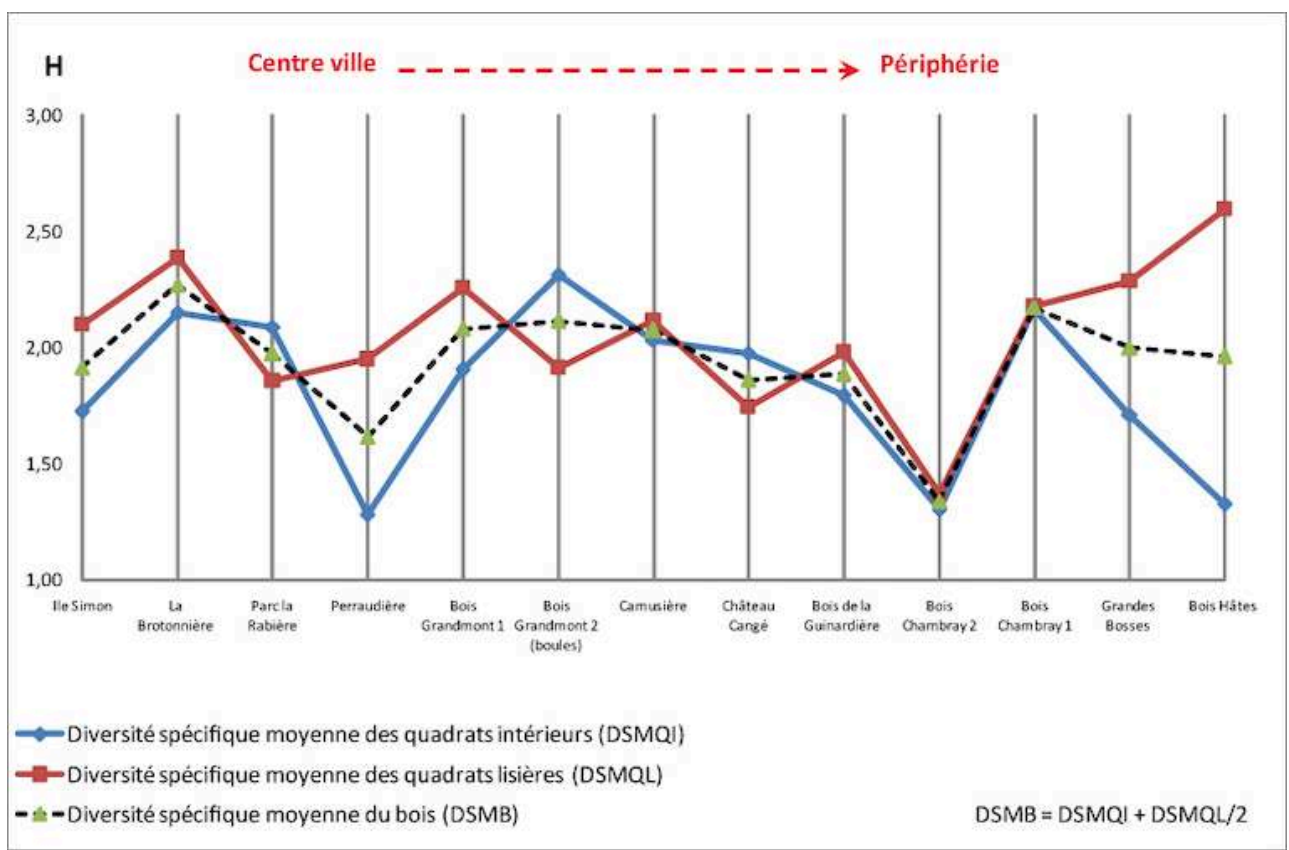

Source : Lotfi Mehdi, 2010.

L'analyse de la végétation des sous-bois (AFC sur les 132 espèces de 110 quadrats dans 11 espaces publics boisés, après exclusion de deux bois particuliers, l'un situé dans le corridor fluvial ligérien et l'autre étant fortement embroussaillé) permet d'identifier les gradients de végétation suivants. Le principal gradient (axe 1) oppose la plupart des espèces ornementales (comme Cyclamen hederifolium Aiton, le cyclamen de Naples, et des variétés horticoles de frênes, tilleuls, pruniers, buis, houx), à la fois d'ombre (sciaphiles) et de lumière (héliophiles), et situées dans les bois centraux, aux espèces non horticoles situées dans les bois périphériques (comme Melampyrum pratense L., Deschampsia flexuosa L., Acer campestre L., Quercus petraea [Matt.] Liebl.). Le deuxième gradient (axe 2) isole des espèces de lumière (comme Dactylis glomerata, Festuca heterophylla), situées dans des bois de grande taille et plus anciens, à la fois centraux et périphériques : c'est un gradient de densité des boisements (figure 4). 
Figure 4.

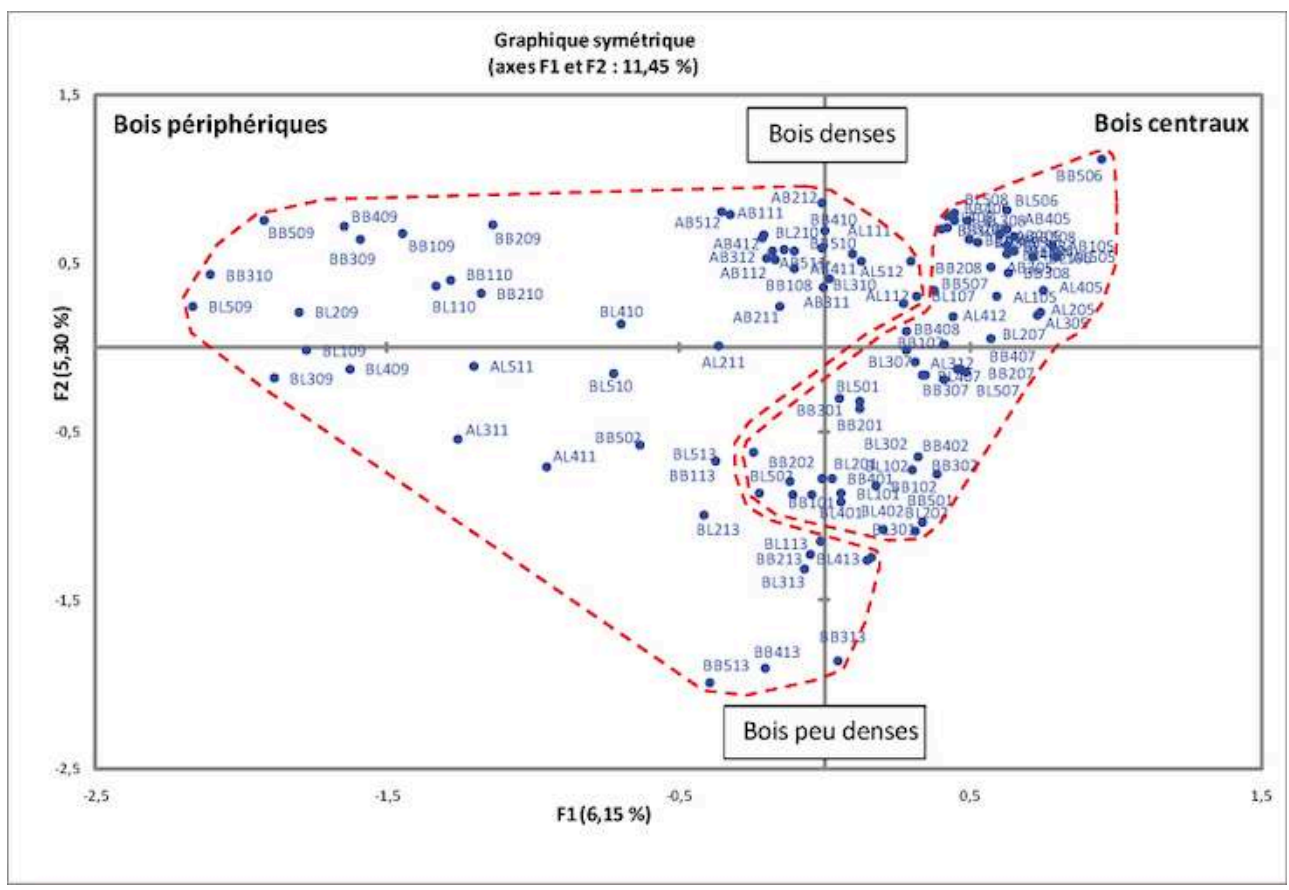

Premier plan (axes 1 et 2) de l'analyse factorielle des correspondances de la composition botanique des bois urbains étudiés (132 espèces $x 110$ quadrats) : relevés identifiant les bois centraux (ensemble de droite) et les bois périphériques (ensemble de gauche).

Source : Lotfi Mehdi, 2010.

14 Si les bois périphériques n'ont pas une diversité spécifique supérieure à celle des bois centraux, comme montré plus haut (figure 3), nous remarquons qu'ils sont en revanche plus dispersés sur le plan factoriel (figure 4), ce qui indique une plus grande variabilité de la composition floristique et, a contrario, une plus grande homogénéité de la végétation des bois centraux.

L'analyse canonique montre que les principales variables corrélées avec la composition botanique des sous-bois sont les parts de surface forestière et de surface urbanisée dans les zones adjacentes aux bois, aux trois distances testées (en ordre croissant). À ces variables suivent les autres : surface du bois ; distances au centre-ville, au cours d'eau et à l'espace vert le plus proche; parts de surfaces agricole et aquatique dans les espaces adjacents; ancienneté du bois. Globalement la position du relevé en zone centrale ou périphérique du bois joue un rôle mineur sur la composition botanique des sous-bois.

\section{Discussion}

Nos recherches sur la flore associée aux arbres dans les paysages urbains d'une agglomération de taille moyenne confirment le lien entre l'occupation du sol adjacente et la composition botanique des bois urbains, ce qui a été montré dans des zones urbaines de plus grande taille (Guirado et al., 2006). Nous soulignons surtout la relation entre la forte proportion de surface urbanisée et la présence d'espèces horticoles (Borgmann et Rodewald, 2005), bien que les espèces horticoles restent marginales dans les sous-bois urbains, comme déjà observé (Vallet et al., 2010). En effet les bois entourés 
par une proportion élevée de surface urbanisée sont aussi ceux qui abritent des espèces horticoles, ce qui est probablement en relation avec la dispersion d'espèces à valeur ornementale en provenance des jardins urbains, publics ou privés. Ces espèces horticoles contribuent à la richesse spécifique élevée des habitats situés dans les centres urbains, comme observé dans d'autres régions (Wania et al., 2006), y compris dans des habitats boisés (Kowarik et al., 2013). En effet, deux éléments expliquent la richesse spécifique élevée de la flore des centres urbains : la forte hétérogénéité des habitats urbains, constitués d'une mosaïque d'habitats originaux et semi-naturels résiduels permettant des conditions écologiques diversifiées favorables à de nombreuses espèces; et la présence d'espèces introduites (Kowarik, 2011). Si des espèces invasives comme l'ailante (Ailanthus altissima [Mill.] Swingle) peuvent induire un appauvrissement des communautés de sous-bois (Motard et al., 2011), l'impact des espèces introduites doit être évalué à plusieurs échelles; nos résultats ne permettent donc pas de confirmer, à cette échelle intra-urbaine, que le milieu urbain homogénéise la flore (McKinney, 2006).

17 Il est ici opportun de préciser que, dans les zones urbaines, la distance au centre-ville n'est pas toujours proportionnelle à la part de surface urbanisée ; en effet des éléments géographiques comme la présence de corridors fluviaux et de zones inondables et donc non constructibles qui y sont associées peuvent, davantage que la distance au centreville, influencer la part de surface urbanisée. C'est le cas dans l'agglomération tourangelle et dans bien des villes fluviales où la tache urbaine ne présente pas une structure radioconcentrique. Le lien entre paysage urbain et végétation forestière doit donc être évalué à l'aune de ces deux aspects distincts du gradient d'urbanisation, comme suggéré dans des travaux d'écologie urbaine (McDonnell et Hahs, 2009).

Notre étude confirme également que les lisières des bois représentent un intérêt certain pour la biodiversité urbaine. Les lisières forestières urbaines sont plus riches en espèces que les zones centrales des bois mais leur composition botanique n'est pas principalement en relation avec cette position dans le bois: d'autres facteurs, et notamment le paysage urbain, jouent un rôle plus important, comme indiqué plus haut et observé aussi dans d'autres études conduites dans des agglomérations de taille comparable (Vallet et al., 2010).

Outre les facteurs spatiaux à l'échelle du paysage (bois centraux/bois périphériques) et de l'habitat (centre/lisière des bois), notre étude montre que des facteurs locaux comme la surface et l'âge du bois jouent également un rôle sur la flore abritée par les bois urbains. Ceci montre que les bois de grande taille - impliquant la possibilité d'une variation de la densité du couvert forestier - apportent une contribution particulière, marquée par des espèces de lumière, à la diversité de la végétation forestière en zone urbaine.

Ces éléments suggèrent l'importance de planifier l'aménagement et la gestion des espaces boisés à plusieurs échelles, de la tache urbaine à l'habitat, et d'introduire les questions relatives à la gestion des arbres en ville dans le contexte de la trame verte urbaine (Clergeau et Blanc, 2013). Sur le plan méthodologique, nos observations confirment également que les indices basés sur la richesse spécifique sont moins sensibles que la composition botanique à la diversité des habitats urbains adjacents (Hobbs, 1988). 


\section{Conclusion}

21 La végétation du sous-bois dans les zones urbaines est donc liée au gradient d'urbanisation et à l'occupation adjacente aux espaces boisés, en particulier à la proportion de surfaces urbanisées; elle est susceptible d'être influencée par la planification urbaine, à travers la localisation réciproque des zones bâties et des espaces boisés. Cette flore est influencée également par les pratiques de gestion des arbres urbains, à toutes les échelles, des arbres d'alignement aux îlots boisés des parcs urbains (Alvey, 2006; Young, 2010). Ceci doit être apprécié dans le contexte du développement du concept de gestion forestière urbaine, urban forestry (Konijnendijk, 2003), qui se développe dans le cadre du changement des objectifs de la plantation et de la gestion des arbres en ville. Ces objectifs dépassent les raisons esthétiques et symboliques traditionnellement associées à la végétation urbaine (Mehdi et al., 2012) et, après avoir été marqués par des fonctions liées aux loisirs tout au long des années 1970 et 1980, s'orientent, depuis les années 1990, vers des fonctions écologiques (Rydberg et Falck, 2000). Ce changement d'objectifs implique l'inclusion de la végétation spontanée comme un élément d'une stratégie de gestion forestière urbaine qui s'appuie sur les processus de colonisation de la végétation semi-naturelle se déroulant à l'échelle du paysage, et en lien avec différents types d'espaces verts publics planifiés (Millard, 2000 ; Millard, 2008).

Les auteurs remercient les deux relecteurs anonymes pour leurs remarques constructives.

\section{BIBLIOGRAPHIE}

Alvey, A., « Promoting and preserving biodiversity in the urban forest », Urban Forestry \& Urban Greening, vol. 5, n² 4, 2006, p. 195-201.

Armson, D., Stringer, P., Ennos, A. R., « The effect of street trees and amenity grass on urban surface water runoff in Manchester, UK », Urban Forestry \& Urban Greening, vol. 12, $n^{\circ}$ 3, p. $282-$ 286.

Bajsanski, I., Stojakovic, V., Jovanovic, M., « Effect of tree location on mitigating parking lot insolation », Computers Environment, Urban Systems, vol. 56, 2016, p. 59-67.

Bennour-Azooz, M., Donadieu, P., Bettaîeb, T., « L'arbre à Tunis : hypothèses pour une histoire de l'espace public ", Projets de paysage, $\mathrm{n}^{\circ} 8$, juillet 2012, URL : http://www.projetsdepaysage.fr/fr/ 1_arbre_tunis_hypoth_ses_pour_une_histoire_de_l_espace_public

Borgmann, K. L., Rodewald, A. D., « Forest Restoration in Urbanizing Landscapes: Interactions Between Land Uses and Exotic Shrubs », Restoration Ecology, vol. 13, n 2, 2005, p. 334-340.

Brun, M., « Biodiversité végétale et délaissés dans l'aménagement urbain. Contribution potentielle des délaissés urbains aux continuités écologiques, thèse de doctorat, université François Rabelais » 2015, 432 p. 
Carreiro, M. M., Tripler, C. E., « Forest Remnants Along Urban-Rural Gradients: Examining Their Potential for Global Change Research », Ecosystems, vol. 8, n 5, 2005, p. 568-582.

Clergeau, P. et Blanc, N., Trames vertes urbaines : de la recherche scientifique au projet urbain, Paris, Le Moniteur, 2013.

Clergeau, P., Jokimäki, J., Snep, R., « Using hierarchical levels for urban ecology ", Trends in Ecology Evolution, vol. 21, n 12, 2006, p. 659-601.

Cowett, F.D., Bassuk, N.L., « Statewide assessment of street trees in New York State, USA », Urban Forestry \& Urban Greening, vol. 13, n². 2014, p. 213-220.

Croci, S., Butet, A., Georges, A., Aguejdad, R., Clergeau, P., « Small urban woodlands as biodiversity conservation hot-spot: a multi-taxon approach ", Landscape Ecology, vol. $23, \mathrm{n}^{\circ} 10$, 2008, p. 1171-1186.

Dornier, A., Pons, V., Cheptou, P.O., « Colonization and extinction dynamics of an annual plant metapopulation in an urban environment ", Oikos, vol. 120, n 8, 2011, p. 1240-1246.

Dubois, J., Cheptou, P.-O., « Effects of fragmentation on plant adaptation to urban environments ", Philosophical Transactions of the Royal Society B, Biological Sciences, 2016, URL : http://rstb.royalsocietypublishing.org/content/372/1712/20160038

Godefroid, S., Koedam, N., « Distribution pattern of the flora in a peri-urban forest: an effect of the city-forest ecotone ", Landscape and Urban Planning, vol. 65, n 4, 2003, p. 169-185.

Grote, R., Samson, R., Alonso, R., Amorim, J.H., Carinanos, P., Churkina, G., Fares, S., Thiec, D. Le, Niinemets, U., Mikkelsen, T.N., Paoletti, E., Tiwary, A., Calfapietra, C., « Functional traits of urban trees: air pollution mitigation potential », Frontiers in Ecology and the Environment, vol. 14, $\mathrm{n}^{\circ} 10$, 2016, p. 543-550.

Guirado, M., Pino, J., Roda, F., « Understorey plant species richness and composition in metropolitan forest archipelagos: effects of forest size, adjacent land use and distance to the edge ", Global Ecolgy \& Biogeography, vol. 15, n², 2006, p. 50-62.

Haddad, Y., « Les arbres d'alignement urbains. Un enjeu pour des partenaires multiples », Les Annales de la recherche urbaine, vol. 75, n 1, 1997, p. 113-118.

Hedblom, M., Söderström, B., « Woodlands across Swedish urban gradients: Status, structure and management implications ", Landscape \& Urban Planning, vol. 84, $n^{\circ}$ 1, 2008, p. 62-73.

Hobbs, E. R., « Species richness of urban forest patches and implications for urban landscape diversity », Landscape Ecology, vol. 1, n 3, 1988, p. 141-152.

Jim, C. Y., « Impacts of intensive urbanization on trees in Hong Kong ", Environmental Conservation, vol. 25, n 2, 1998, p. 146-159.

Kong, F. et Nakagoshi, N., « Spatial-temporal gradient analysis of urban green spaces in Jinan, China », Landscape \& Urban Planning, vol. 78, $\mathrm{n}^{\circ}$ 3, 2006, p. 147-164.

Kong, F., Nobukazu, N., Yin, H., Akira, K., « Spatial gradient analysis of urban green spaces combined with landscape metrics in Jinan City of China », Chinese Geographical Science, vol. 15, $\mathrm{n}^{\circ} 2,2005$, p. 254-261.

Konijnendijk, C.C., « A decade of urban forestry in Europe », Forest Policy and Economics, vol. 5, $n^{\circ}$ 2, 2003, p. 173-186.

Kowarik, I., « Novel urban ecosystems, biodiversity, and conservation », Environmental Pollution, vol. 159, n 8-7, 2011, p. 1974-1983. 
Kowarik, I., Lippe, M. von der, Cierjacks, A., « Prevalence of alien versus native species of woody plants in Berlin differs between habitats and at different scales ", Preslia, vol. 85, n² 2, 2013, p. 113-132.

McDonnell, M.J., Hahs, A. K., « Comparative ecology of cities and towns: past, present and future ", dans McDonnell, M. J., Hahs, A. K., Breuste, J. H. (ed.), Ecology of Cities and Towns: A Comparative Approach, Cambridge, Cambridge University Press, 2009, 71-89 p.

McKinney, M. L., « Urbanization as a major cause of biotic homogenization », Biological Conservation, vol. 127, $\mathrm{n}^{\circ} 3,2006$, p. 247-260.

McPherson, E. G., Simpson, J. R., « Reducing Air Pollution through Urban Forestry. Can Parking Lot Trees Improve Air Quality?», 48th Annual Meeting of the California Forest Pest Council, 1999, p. 48-51.

Mehdi, L., Weber, C., Di Pietro, F., Selmi, W., « Évolution de la place du végétal dans la ville, de l'espace vert à la trame verte », Vertigo, vol. 12, n², 2012, URL : https://vertigo.revues.org/ 12670.

Mehdi, L., « Structure verte et biodiversité urbaine. L'espace vert: analyse d'un écosystème anthropisé », thèse de doctorat, université François Rabelais, 2010, 397 p.

Millard, A., " Semi-natural vegetation and its relationship to designated urban green space at the landscape scale in Leeds, UK », Landscape Ecology, vol. 23, n 10, 2008, p. 1231-1241.

Millard, A., « The potential role of natural colonisation as a design tool for urban forestry. A pilot study ", Landscape and Urban Planning, vol. 52, 2000, p. 173-179.

Motard, E., Muratet, A., Clair-Maczulajtys, D., Machon, N., « Does the invasive species Ailanthus altissima threaten floristic diversity of temperate peri-urban forests? », Comptes Rendus Biologies, vol. 334, n 12, 2011, p. 872-879.

Mullaney, J., Lucke, T., Trueman, S. J., « A review of benefits and challenges in growing street trees in paved urban environments », Landscape and Urban Planning, vol. 134, 2015, p. 157-166.

Nitoslawski, S., Duinker, P., Bush, P., « A review of drivers of tree diversity in suburban areas: Research needs for North American cities ", Environmental Reviews, vol. 24, n 4, 2016, p. 471-483.

Pellegrini, P., « Pieds d'arbre, trottoirs et piétons: vers une combinaison durable?», Développement durable \& Territoires, vol. 3, $\mathrm{n}^{\circ} 2,2012$, URL : https:// developpementdurable.revues.org/9329.

Pirnat, J., « Conservation and management of forest patches and corridors in suburban landscapes ", Landscape \& Urban Planning, vol. 52, n 2-3, 2000, p. 135-143.

Qvistrom, M., " The nature of running: On embedded landscape ideals in leisure planning ", Urban Forestry \& Urban Greening, vol. 17, 2016, p. 202-210.

Rydberg, D., Falck, F., « Urban forestry in Sweden from a sylvicultural perspective: a review », Landscape \& Urban Planning, vol. 47, $\mathrm{n}^{\circ}$ 1-2, 2000, p. 1-18.

Santamaria, F., « La notion de "ville moyenne" en France, en Espagne et au Royaume-Uni//The notion of "medium-sized town" in France, Spain and the United Kingdom », Annales de Georgraphie, vol. 109, n 613, 2000, p. 227-239.

Säumel, I., Weber, F., Kowarik, I., « Toward livable and healthy urban streets: Roadside vegetation provides ecosystem services where people live and move », Environmental Science \& Policy, vol. 62, 2015, p. 24-33. 
Skärbäck, E., « Urban forests as compensation measures for infrastructure development », Urban Forestry \& Urban Greening, vol. 6, n 4, 2007, p. 279-285.

Sonntag-Öström, E., Stenlund, T., Nordin, M., Lundell, Y., Ahlgren, C., Fjellman-Wiklund, A., Järvholm, L. S., Dolling, A., « Nature's effect on my mind” - Patients' qualitative experiences of a forest-based rehabilitation programme », Urban Forestry \& Urban Greening, vol. 14, n 3, 2015, p. 607-614.

Subburayalu, S., Sydnor, T. D., « Assessing street tree diversity in four Ohio communities using the weighted Simpson index ", Landscape and Urban Planning, vol. 106, $\mathrm{n}^{\circ} 1,2012,44-50$, URL: http://doi.org/10.1016/j.landurbplan.2012.02.004.

Tollis, C., « Pour le meilleur et pour le pire ! Les arbres en ville peuvent-ils faire patrimoine? Analyse des spatialités concurrentes arbres-riverains à Grenoble », Vertigo, hors série 16, juin 2013, URL : https://vertigo.revues.org/13736.

Vallet, J., Beaujouan, V., Pithon, J., Rozé, F., Daniel, H., « The effects of urban or rural landscape context and distance from the edge on native woodland plant communities ", Biodiversity and Conservation, vol. 19, $\mathrm{n}^{\circ} 12,2010$, p. 3375-3392.

Vallet, J., «Gradient d'urbanisation et communautés végétales d'espaces boisés. Approche à plusieurs échelles dans trois agglomérations du Massif armoricain », thèse de doctorat, université d'Angers, 2009, $258 \mathrm{p}$.

Voisin, L., «La mobilisation du paysage par les acteurs publics locaux, un enjeu stratégique de territorialisation? Réflexions en Loire Moyenne : Blois, Nevers, Saumur ", thèse de doctorat, université François Rabelais, 2013, 415 p.

Wania, A., Kühn, I., Klotz, S., « Plant richness patterns in agricultural and urban landscapes in Central Germany - Spatial gradients of species richness ", Landscape \& Urban Planning, vol. 75, $\mathrm{n}^{\circ}$ 1-2, 2006, p. 97-110.

Weber, F., Kowarik, I., Säumel, I., « Herbaceous plants as filters: Immobilization of particulates along urban street corridors », Environmental Pollution, vol. 186, 2014a, p. 234-240.

Weber, F., Kowarik, I., Säumel, I., « A walk on the wild side: Perceptions of roadside vegetation beyond trees », Urban Forestry \& Urban Greening, vol. 13, n², 2014b, p. 205-212.

White, J. G., Antos, M. J., Fitzsimons, J. A., Palmer, G. C., « Non-uniform bird assemblages in urban environments: The influence of streetscape vegetation ", Landscape \& Urban Planning, vol. 71, $n^{\circ} 2-4,2005$, p. 123-135.

Wittig, R., Becker, U., « The spontaneous flora around street trees in cities-A striking example for the worldwide homogenization of the flora of urban habitats », Flora-Morphology, Distribution, Functional Ecology of Plants, vol. 205, n 10, 2010, p. 704-709.

Young, R. F., « Managing municipal green space for ecosystem services », Urban Forestry \& Urban Greening, vol. 9, n 4, 2010, p. 313-321.

\section{ANNEXES}

Tableau 2. Liste des espèces trouvées dans les sous-bois étudiés

\begin{tabular}{|l|l|}
\hline Genres - espèces & Genres - espèces \\
\hline
\end{tabular}




\begin{tabular}{|c|c|}
\hline Acer campestre L. & Conopodium majus (Gouan) Loret \\
\hline Acer palmatum Thunb. & Convolvulus arvensis $L$. \\
\hline Acer platanoides $L$. & Conyza canadensis (L.) Cronquist \\
\hline Acer pseudoplatanus L. & Cornus mas L. \\
\hline Acer saccharinum $L$. & Cornus sanguinea L. \\
\hline Achillea millefolium L & Corylus avellana $L$. \\
\hline Aesculus turbinata & Crataegus laevigata (Poir.) DC. \\
\hline Agrimonia eupatoria $L$. & Crataegus monogyna Jacq. \\
\hline Agrostis capillaris L. & Crepis capillaris (L.) Wallr. \\
\hline Agrostis capillaris subsp. capillaris & Crepis setosa Haller $f$. \\
\hline Agrostis clavata & Cyclamen hederifolium Aiton \\
\hline Agrostis stolonifera L. & Cytisus scoparius (L.) Link \\
\hline Ailanthus altissima (Mill.) Swingle & Dactylis glomerata $L$. \\
\hline Ajuga reptans $L$. & Danae racemosa (L.) Moench \\
\hline Alliaria petiolata (M. Bieb.) C \& G & Daphne laureola $L$. \\
\hline Alopecurus myosuroides Huds. & Daucus carota $L$. \\
\hline Anagallis arvensis $L$. & Deschampsia flexuosa (L.) Trin. \\
\hline Andryala integrifolia $L$. & Digitaria sanguinalis (L.) Scop. \\
\hline Anthemis arvensis $L$. & Epilobium tetragonum L. \\
\hline Arenaria serpyllifolia L. & Epipactis atrorubens (Hoffm.) Besser \\
\hline Arrhenatherum elatius (L.) P.Beauv. & Erodium cicutarium (L.) L'Hér. \\
\hline Arum italicum Mill. & Euonymus europaeus L. \\
\hline Arum maculatum $L$. & Euonymus japonicus L.f. \\
\hline Bellis perennis $L$. & Euphorbia amygdaloides $L$. \\
\hline Blackstonia perfoliata (L.) Huds. & Fagus sylvatica $L$. \\
\hline Brachypodium sylvaticum (Huds.) P.Beauv. & Festuca heterophylla Lam. \\
\hline Bromus hordeaceus ssp hordeaceus & Festuca rubra L. \\
\hline
\end{tabular}




\begin{tabular}{|c|c|}
\hline Buxus sp (hort) & Fragaria vesca $L$. \\
\hline Calluna vulgaris (L.) Hull & Fraxinus excelsior $L$. \\
\hline Caprinus betulus & Fraxinus ornus $L$. \\
\hline Carex flacca Schreb. & Fraxinus sp (hort) \\
\hline Carex sylvatica Huds. & Galium mollugo L. \\
\hline Castanea sativa Mill. & Galium uliginosum $L$. \\
\hline Centaurea jacea $L$. & Geranium dissectum $L$. \\
\hline Centaurea nigra $L$. & Geranium robertianum $L$. \\
\hline Centaurium erythraea Raf. & Geum rivale $L$. \\
\hline Cerastium fontanum subsp. Vulgare & Geum urbanum $L$. \\
\hline Cerastium glomeratum Thuill. & Glechoma hederacea $L$. \\
\hline Cercidiphyllum japonicum & Hedera helix $L$. \\
\hline Cirsium arvense (L.) Scop. & Heracleum sphondylium $L$. \\
\hline Cirsium dissectum (L.) Hill & Hieracium murorum $L$. \\
\hline Cirsium vulgare (Savi) Ten. & Hieracium sabaudum $L$. \\
\hline Clematis vitalba $L$. & Hieracium umbellatum $L$. \\
\hline Clinopodium vulgare $L$. & Holcus lanatus $L$. \\
\hline Coneaster horizontalis & Hordeum murinum $L$. \\
\hline Hypericum hirsutum L. & Poa annua $L$. \\
\hline Hypericum perforatum $L$. & Poa nemoralis $L$ \\
\hline Hypericum pulchrum $L$. & Poa pratensis $L$. \\
\hline Hypochaeris radicata $L$. & Polygonatum multiflorum (L.) All. \\
\hline Ilex aquifolium $L$. & Polygonum aviculare $L$. \\
\hline Ilex sp (hort) & Polygonum persicaria L. \\
\hline Inconnue sp1 & Populus alba $L$. \\
\hline Inconnue sp2 (hort) & Potentilla erecta (L.) Räusch. \\
\hline Juglans regia L. & Potentilla reptans $L$. \\
\hline
\end{tabular}




\begin{tabular}{|c|c|}
\hline Juncus acutiflorus Ehrh. ex Hoffm. & Primula veris $L$. \\
\hline Lactuca serriola L. & Prunella vulgaris $L$. \\
\hline Lamium galeobdolon subsp. galeobdolon & Prunus avium (L.) L. \\
\hline Lamium maculatum (L.) L. & Prunus laurocerasus L. \\
\hline Lapsana communis $L$. & Prunus sp (hort) \\
\hline Lathyrus linifolius subsp. montanus & Prunus spinosa $L$. \\
\hline Lathyrus pratensis $L$. & Prynus sp (hort) \\
\hline Ligustrum vulgare L. & Pteridium aquilinum (L.) Kuhn \\
\hline Logfia minima (Sm.) Dumort. & Pulmonaria longifolia (Bastard) Boreau \\
\hline Lolium perenne L. & Pyracantha pauciflora (Poir.) M.Roem. \\
\hline Lonicera japonica Thunb. ex Murray & Quercus ilex $L$. \\
\hline Lonicera nitida E.H.Wilson & Quercus palustris Münchh. \\
\hline Lonicera periclymenum $L$. & Quercus petraea Liebl. \\
\hline Lotus corniculatus $L$. & Quercus pubescens Willd. \\
\hline Luzula forsteri (Sm.) DC. & Quercus robur $L$. \\
\hline Lysimachia nummularia L. & Ranunculus acris $L$. \\
\hline Magnolia campbellii & Ranunculus bulbosus $L$. \\
\hline Malus sylvestris Mill. & Ranunculus repens $L$. \\
\hline Malva sylvestris $L$. & Ribes rubrum L. \\
\hline Medicago arabica (L.) Huds. & Robinia pseudoacacia L. \\
\hline Medicago lupulina L. & Rosa arvensis Huds. \\
\hline Melampyrum pratense L. & Rubia peregrina $L$. \\
\hline Melica uniflora Retz. & Rubus caesius $L$. \\
\hline Mespilus germanica L. & Rubus fruticosus $L$. \\
\hline Milium effusum L. & Rubus idaeus L. \\
\hline Molinia caerulea (L.) Moench & Rumex acetosa $L$. \\
\hline Mycelis muralis (L.) Dumort. & Rumex acetosella L. \\
\hline
\end{tabular}




\begin{tabular}{|c|c|}
\hline Myosotis ramosissima Rochel & Rumex alpinus $L$. \\
\hline Ophrys apifera Huds. & Rumex obtusifolius L. \\
\hline Orobanche minor Sm. & Rumex palustris Sm. \\
\hline Orobanche purpurea Jacq. & Rumex rupestris Le Gall \\
\hline Oxalis articulata Savigny & Rumex sanguineus $L$. \\
\hline Peucedanum gallicum Latourr. & Rumex thyrsiflorus Fingerh. \\
\hline Picris echioides $L$. & Ruscus aculeatus L. \\
\hline Pinus sylvestris $L$. & Salix cinerea L. \\
\hline Plantago lanceolata L. & Sanicula europaea L. \\
\hline Saponaria officinalis $L$. & Thuja plicata D.Don ex Lamb. \\
\hline Scrophularia nodosa $L$. & Tilia sp1 (hort) \\
\hline Senecio jacobaea L. & Tilia sp2 (hort) \\
\hline Senecio vulgaris $L$. & Tilia x europaea $L$. \\
\hline Serratula tinctoria L. & Torilis japonica (Houtt.) DC. \\
\hline Silene dioica (L.) Clairv. & Trifolium pratense L. \\
\hline Silene vulgaris (Moench) Garcke & Trifolium repens $L$. \\
\hline Solanum dulcamara $L$. & Ulmus laevis Pall. \\
\hline Solidago virgaurea $L$. & Urtica dioica $L$. \\
\hline Sonchus asper (L.) Hill & Vaccinium myrtillus L. \\
\hline Sorbus aucuparia L. & Verbena officinalis $L$. \\
\hline Sorbus domestica L. & Veronica chamaedrys $L$. \\
\hline Sorbus torminalis (L.) Crantz & Veronica officinalis $L$. \\
\hline Stachys officinalis (L.) Trévis. & Viburnum tinus $L$. \\
\hline Stellaria holostea L. & Vicia lathyroides var. lathyroides \\
\hline Stellaria media (L.) Vill. & Vicia sativa $L$. \\
\hline Tamus communis $L$. & Viola hirta $L$. \\
\hline Taraxacum campylodes G.E.Haglund & Viola odorata L. \\
\hline
\end{tabular}




\begin{tabular}{|l|l|}
\hline Taxus baccata L. & Viola reichenbachiana Jord. \\
\hline Teucrium scorodonia L. & \\
\hline
\end{tabular}

hort : espèce horticole.

\section{RÉSUMÉS}

Cet article présente les recherches conduites sur la végétation associée aux arbres dans les espaces boisés urbains, et ses relations avec le paysage, dans une ville moyenne : l'agglomération de Tours. Une étude approfondie de la végétation des bois urbains a permis d'analyser les liens entre cette flore et 1) la structure spatiale urbaine (espaces boisés centraux et périphériques), 2) la structure spatiale interne de ces îlots boisés (zone centrale et de lisière). La structure urbaine joue un rôle prépondérant sur la composition botanique des sous-bois: les espaces boisés centraux (entourés d'une part élevée de surface urbanisée) abritent la quasi-totalité des espèces horticoles présentes, ce qui contribue à leur richesse spécifique. Les bois périphériques, très étendus et entourés majoritairement de cultures, n'ont pas une diversité spécifique supérieure à celle des bois centraux. La part de surface forestière et celle de surface urbanisée dans les zones adjacentes aux espaces boisés, à différents rayons, sont des facteurs majeurs de la composition botanique des bois urbains. En ce qui concerne le lien entre la structure spatiale interne des espaces boisés urbains et la végétation des bois, les lisières des bois ont une diversité spécifique généralement supérieure à celle des zones centrales, bien que les communautés végétales centrales et de lisière soient très similaires. Nos résultats confirment des éléments identifiés dans les grandes agglomérations : la végétation spontanée dans les espaces boisés urbains est liée à la structure du paysage urbain: les stratégies de gestion forestière urbaine devraient prendre en compte les processus de colonisation de la végétation semi-naturelle, se déroulant à l'échelle du paysage.

This article presents research conducted on the vegetation associated with trees in urban wooded areas, and its relationship to the landscape in a medium-sized city: the agglomeration of Tours. An in-depth study of the vegetation of urban wooded areas made it possible to study the links between this flora and 1) the urban spatial structure (central and peripheral wooded areas) and 2) the internal spatial structure of wooded clusters (central and outlying zones). The urban structure plays a dominant role in the botanic compositions of the woodland undergrowth: the central wooded areas (surrounded by a high proportion of urbanised surfaces) harbour almost all the horticultural species present, thus contributing to their diversity. The peripheral woods, extensive and mainly surrounded by cultivated land, do not present a higher degree of diversity compared to the central wooded areas. The proportion of woodland surface area compared with the urbanised surface areas adjacent to wooded areas of varying radiuses, are major factors in the botanic compositions of urban woods. In the interrelation between the internal spatial structure of urban wooded areas and the vegetation of the woods, the outskirts of the woods have a specific diversity which is greater than that of the central zones, even though the plant communities of the central and outlying zones are very similar. Our results confirm elements identified in large agglomerations: spontaneous vegetation in urban wooded spaces is linked to the structure of the urban landscape; urban forestry management strategies should take into account the colonisation process of semi-natural vegetation at the level of the landscape. 


\section{INDEX}

Mots-clés : espaces boisés urbains, structure spatiale urbaine, paysage urbain, sous-bois, végétation spontanée

Keywords : urban wooded areas, urban spatial structure, urban landscape, undergrowth, spontaneous vegetation

\section{AUTEURS}

\section{FRANCESCA DI PIETRO}

Francesca Di Pietro est enseignante-chercheuse à l'université François Rabelais/UMR CITERES (Tours), géographe et écologue du paysage. Elle étudie la végétation des espaces urbains et anthropisés, notamment des espaces de nature en ville et des dépendances vertes. Ses recherches portent sur les caractéristiques et les usages de cette végétation, aux échelles de l'habitat et du paysage.

dipietro[at]univ-tours[dot]fr

http://www.univ-tours.fr/mme-di-pietro-francesca-52764.kjsp

\section{LOTFI MEHDI}

Lotfi Mehdi est docteur en aménagement de l'espace et urbanisme, écologue urbain, membre associé de l'UMR CITERES (Tours) et de l'UMR LIVE (Strasbourg). Il développe des approches des écosystèmes urbains et leurs interactions avec la trame verte, selon deux visions : écocentrique estimation de la connectivité écologique - et anthropocentrique - évaluation des services écosystémiques.

lotfi.mehdi[at]live-cnrs.unistra[dot]fr 